\title{
Evidence-based usability design principles for medication alerting systems
}

\author{
Romaric Marcilly ${ }^{1^{*}} \mathbb{D}$, Elske Ammenwerth ${ }^{2}$, Erin Roehrer ${ }^{3}$, Julie Niès ${ }^{4}$ and Marie-Catherine Beuscart-Zéphir ${ }^{1}$
}

\begin{abstract}
Background: Usability flaws in medication alerting systems may have a negative impact on clinical use and patient safety. In order to prevent the release of alerting systems that contain such flaws, it is necessary to provide designers and evaluators with evidence-based usability design principles. The objective of the present study was to develop a comprehensive, structured list of evidence-based usability design principles for medication alerting systems.

Methods: Nine sets of design principles for medication alerting systems were analyzed, summarized, and structured. We then matched the summarized principles with a list of usability flaws in order to determine the level of underlying evidence.

Results: Fifty-eight principles were summarized from the literature and two additional principles were defined, so that each flaw was matched with a principle. We organized the 60 summarized usability design principles into 6 meta-principles, 38 principles, and 16 sub-principles. Only 15 principles were not matched with a usability flaw. The 6 meta-principles respectively covered the improvement of the signal-to-noise ratio, the support for collaborative working, the fit with a clinician's workflow, the data display, the transparency of the alerting system, and the actionable tools to be provided within an alert.

Conclusions: It is possible to develop an evidence-based, structured, comprehensive list of usability design principles that are specific to medication alerting systems and are illustrated by the corresponding usability flaws. This list represents an improvement over the current literature. Each principle is now associated with the best available evidence of its violation. This knowledge may help to improve the usability of medication alerting systems and, ultimately, decrease the harmful consequences of the systems' usability flaws.
\end{abstract}

Keywords: Human engineering, Usability, Alerting system, Decision support, Design

\section{Background}

Medication alerting systems "provide real-time notification of errors, potential hazards or omissions" related to the prescription of medications, and thus help clinicians to make informed decisions (nota bene: in the present report, a "clinician" is defined as any healthcare professional who interacts with the patient; the term therefore encompasses physicians, nurses and pharmacists) [1]. These promising technologies can change prescribers' behavior by helping them avoid errors [2] and, ultimately, can improve the quality of the medication

\footnotetext{
* Correspondence: romaric.marcilly@univ-lille.fr

'Univ. Lille, INSERM, CHU Lille, CIC-IT / Evalab 1403 - Centre d'Investigation clinique, EA 2694, F-59000 Lille, France, Maison Régionale de la Recherche Clinique, 6 rue du professeur Laguesse, 59000 Lille France

Full list of author information is available at the end of the article
}

management process [3]. Nonetheless, the design and the implementation of these tools may introduce negative, unforeseen side effects: poor integration into the clinical workflow [4], acceptance issues, and decreased safety and quality of care, for example [5]. Some of these issues are related to the usability of the alerting systems [6]; they are caused by defects in the design of the system, i.e. usability flaws. For instance, alerts may be poorly integrated into the workflow and may appear too late in the decision-making process - rendering the alerting system useless $[7,8]$. In other cases, the content of the alert is either incomplete or not visible enough to adequately support a clinician's decision making - leading to incorrect clinical decisions [9]. This lack of information also increases the clinician's cognitive load [10]. Alerts may be poorly written or explained - causing

(c) The Author(s). 2018 Open Access This article is distributed under the terms of the Creative Commons Attribution 4.0 International License (http://creativecommons.org/licenses/by/4.0/), which permits unrestricted use, distribution, and 
misunderstandings or at least creating difficulties in understanding them. These cognitive issues may also lead to incorrect clinical decisions [11-13]. In summary, these and other usability flaws in the alerting system may have severe consequences, such as rejection of the alerting system, and incorrect clinical decisions. Therefore, the usability of an alerting system warrants special scrutiny, with a view to avoiding usability-induced use errors at least.

To prevent the usability of alerting systems from introducing errors, usability activities (e.g. design specifications and prototype evaluation) must be undertaken during the technology development process [14]. The implementation of those activities requires a sound knowledge of good usability design principles (also known as usability heuristics and usability criteria). Violation of those principles may generate usability flaws in the technology. With a view to helping companies to avoid the release of medication alerting systems that contain unintentional violations of these principles, it is necessary to provide designers and evaluators with easy access to relevant, illustrated usability design principles and to convince them of the value of applying these principles to design decisions. In summary, designers and evaluators of medication alerting systems need to access evidence-based usability design principles, i.e. usability design principles that have proven their value in practice [15]. As far as we know, the present study is the first to have provided evidence-based usability design principles for medication alerting systems.

Putting together a body of evidence relies on the accumulation of results that demonstrate the positive value of applying design principles. Unfortunately, publications in the field of usability evaluation tend to report only negative results, i.e. instances of usability flaws. This reporting bias prevents the collection of evidence to show that applying principles is beneficial. Hence, although it is not yet possible to demonstrate the positive value of applying usability design principles, it is still possible to demonstrate the negative consequences of violating them.

In previous research, we started to develop a usability knowledge framework (Fig. 1; [16]). We have used this framework to gather evidence-based usability design principles for medication alerting systems. In a first step, we performed a systematic review of the literature to identify the usability flaws in medication alerting systems used in hospital and/or primary care (active or passive alerts, and use as a standalone system or integrated into a larger information system) [17]. In a second step, we searched for the consequences of these flaws on users (usage problems; e.g. alert fatigue and missed information) and on the work system (negative outcomes; e.g. a decrease in effectiveness, and patient safety issues), and linked them to their cause [6].

The third step involves identifying, summarizing, and organizing published design principles so as to avoid "reinventing" principles as far as possible. The fourth step (in line with previous work by Nielsen [18]) seeks to match usability flaws to the usability design principles that could fix them and thus obtain empirical illustrations of the principles' violation. The present study tackled the third and fourth steps. The results will help to establishing evidence in support of these principles.

The present study had two objectives. Firstly, it sought to identify and organize literature reports of usability design principles for medication alerting systems in hospital or primary care settings into a comprehensive, structured list of design principles. Secondly, the study sought to match this list with the set of usability flaws identified in the systematic review [17], in order to assess the fit between known usability flaws and known existing design principles and thus illustrate violations of these principles.

\section{Methods}

A two-step methodology was applied.

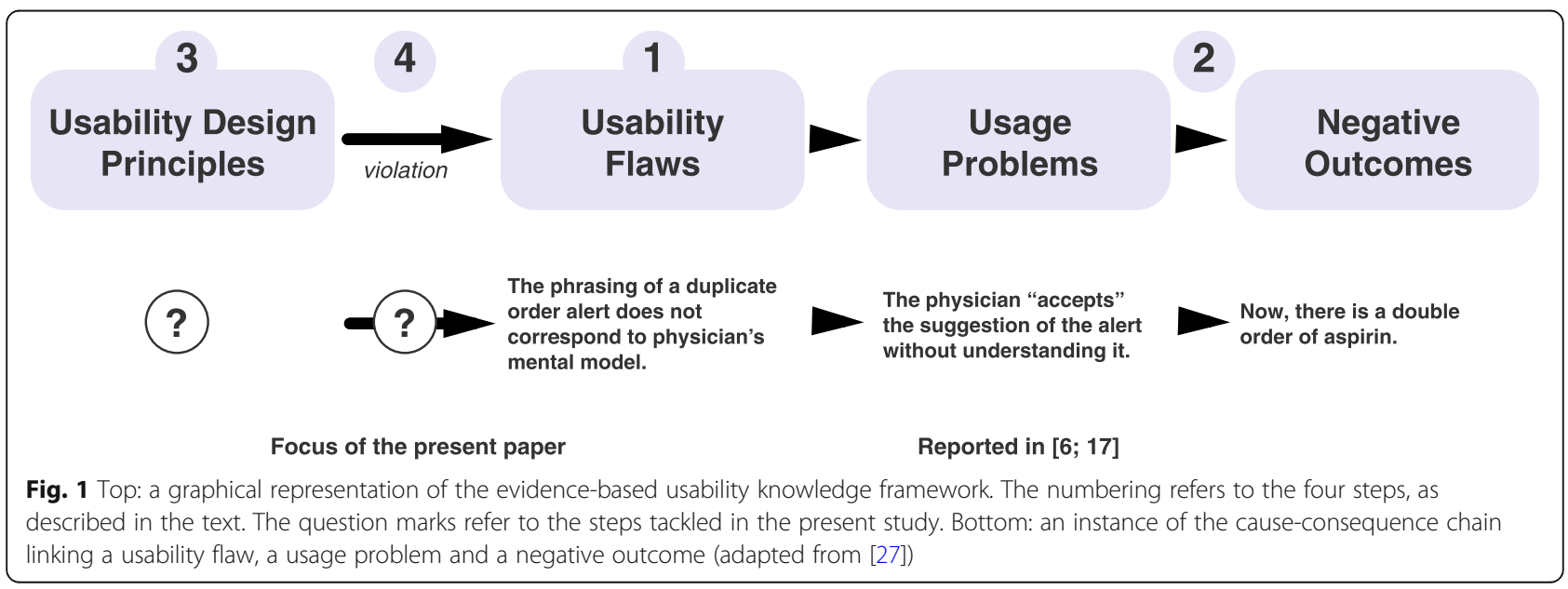




\section{Gathering and structuring usability design principles}

We searched peer-reviewed journals and conference papers for published consensus sets of usability design principles for medication alerting systems (i.e. principles that experts in the field had agreed on). The "grey" literature was excluded because the quality of the information may vary. Hence, we searched PubMed, Scopus, and Ergonomics Abstracts databases for articles addressing both "medication alerting systems" and "usability" topics. With this goal in mind, we used the screening and eligibility assessment steps from our previous systematic review [17] to identify papers purposefully providing at least one usability design principle dedicated to medication alerting systems. We excluded system-specific papers providing recommendations on improving usability because these principles are not applicable to a broad range of systems. This task was updated on March 30th 2016. The literature search was intended to provide an overview of published sets of usability design principles for medication alerting systems, rather than being systematic and reproducible. The database search was completed by examining the investigators' personal libraries and by screening the references of the selected publications. Two investigators (MCBZ and RM) decided on the final list of publications by consensus.

Once relevant publications had been identified, one investigator (RM) extracted all items referring to usability design principles from each publication. Next, the investigator grouped together principles with similar purposes and organized them hierarchically. A second investigator (MCBZ) independently crosschecked the hierarchical organization of the principles. Disagreements were solved by discussion until a consensus was reached. Lastly, the two investigators summarized the principles that had been grouped together.

\section{Matching usability design principles to known usability flaws}

One investigator (RM) checked the list of usability flaws published in the on-line appendices of Marcilly et al. [17] against the structured list of usability design principles. A second investigator (MCBZ) crosschecked the results. Disagreements were discussed until a consensus was reached. The items referring to usability flaws were either descriptions of the technology's defects observed during field studies or usability tests, answers to interviews/questionnaires, or users' positive or negative comments about the characteristics of the technology collected during their interaction with the system. A usability flaw was matched to a given usability design principle if it was an instance of a violation of the said principle. Reciprocally, a usability design principle matched a usability flaw if the application of the principle stopped the flaw from occurring. If a flaw did not match any of the usability design principles, then we considered the possible extension (broadening) of an existing principle to other contexts so that it covered a wider range of flaws. If no principles could be extended to cover the flaw, we defined a new principle.

The matching process was intended to be as unequivocal as possible, i.e. one flaw matched one principle. However, if a given usability flaw violated several principles (e.g. at different levels of granularity), we matched that flaw to the most significantly violated principles (based on our experience). It should be noted that a given principle could be matched with several instances of the same flaw.

Figure 2 illustrates the matching process. Both investigators performed the descriptive analysis of the matches.

\section{Results}

Gathering and structuring usability design principles

We identified 9 publications on design principles dedicated to medication alerting systems (Table 1).

Figure 3 describes the sets of publications analyzed. One publication (Zachariah et al. [22]) was included in both sets; although this publication was an extension of another set of design principles described by Phansalkar et al. [20], it contained a few usability design principles not found in the original publication [20]. The publication also gave a list of usability flaws detected using heuristics. Despite the potential for self-matching bias, this publication was included because our objective was to obtain the most comprehensive possible list of design principles. Moreover, it was found that virtually all first authors of the set of usability design principles were co-authors of one or more studies included in the review of usability flaws (e.g.[1, 4, 20, 23]).

A total of 345 items referring to usability design principles were extracted from the 9 publications (see Additional file 1: Appendix 1) and then organized. The level of agreement between the two investigators regarding the organization of the items was very high, with full agreement for $92.6 \%$ of the combinations, discussion needed for $6 \%$, and disagreement for $1.3 \%$. After a consensus meeting, the items were summarized into 58 principles. No significant inconsistencies between principles from different publications were noticed. The summarized principles displayed different granularity levels, and some were more tangible and precise than others; they could therefore be organized hierarchically into 6 meta-principles, 36 principles, and 16 sub-principles (Fig. 4, Table 2).

Overall, the 9 publications contributed to different extents to the set of summarized principles: the contributions ranged from 12\% [22] to 69\% [24] (see Additional file 2: Appendix 2).

The level of support for each of the summarized principles (in terms of number of publications in which they were found) varied: one summarized principle was supported by all 9 papers (\#49 "Include actionable tools 


\section{Usability Design Principles}

Meta-principle E: (\#44) Make the system transparent for the user. The system must not be a black-box and its coverage must be accessible to its user [19]. Inform users when necessary data are missing (e.g. severity of the unsafe event) [24].

Make accessible:

\#48 The events that are checked by the alerting system [20] ...

... and the type and format of data (e.g. freetext, origin of the data (other hospital data), name of the drugs vs. ATC codes).

\section{Corresponding Usability Flaws}

The decision support is invisible to users [42]: capabilities and limitations of the system along with types of data that are checked are not shown to the users $[10 ; 27 ; 42]$

\section{Corresponding Usability Flaws}

The system does not explain which interactions are actually checked [10], ...

... which patient's data are analyzed [31], whether orders in free text are checked [10] and whether checking is based on drugs' names or on ATC codes [13]

Fig. 2 Illustration of the matching process, using meta-principle E (\#44) and one of its sub-principles (\#48). The usability design principles found in the literature were summarized and organized hierarchically (left). The usability flaws identified in the systematic review were collated by topic (right). Next, the correspondence between a given type of flaw and a given summarized principle was established based on the principle's ability (if applied) to fix the usability flaw. This correspondence is represented by a double arrow. When a usability flaw could not be fixed by any of the design principles in the literature, we either extended an existing principle or created a new one (single arrow). The illustration presents an extension of principle \#48 (in italics)

within the alert"), "Suggest, do not impose" (\#42) was supported by 8 publications, and 6 principles were found in 1 publication. When considering the overall meta-principles and their components (i.e. related principles and sub-principles), the level of support ranged from 5 publications for meta-principle E ("Make the system transparent for the user") to 9 publications for meta-principle D ("Display relevant data within the alert") and meta-principle F ("Include actionable tools within the alert").

\section{Matching usability design principles with known usability flaws}

The two investigators agreed well on the matching between usability flaws and usability design principles, with full agreement for $54.6 \%$ of matches, partial agreement for $31 \%$ (agreement on the main corresponding principle but a need to match the flaw with a second principle), discussion needed for $13.4 \%$, and disagreement for $1 \%$. Of the 58 principles, 34 directly matched at least one instance of a usability flaw, nine were broadened to cover a flaw, and 15 were not matched at all (see Additional file 3: Appendix 3). Two new principles were defined so that all flaws matched a principle (\#46, provide "a description of the characteristics of the tools included in the alert" to users, and \#57, include a "send the alert into the clinical note template" function in the alert).

After the addition of the 2 new principles, the final set comprised 60 summarized usability design principles: 6 meta-principles, 38 principles, and 16 sub-principles. The 6 meta-principles were as follows:

Table 1 Main characteristics of the publications on usability design principles

\begin{tabular}{|c|c|c|c|c|}
\hline & First author & Year & Focus & Method used to provide the principles \\
\hline [2] & Bates DW & 2003 & Design, implementation, monitoring & Lessons learned \\
\hline [19] & Kuperman GJ & 2007 & Design, implementation & Expert consensus \\
\hline [4] & Sittig DF & 2008 & Design, implementation, research & Lessons learned / expert consensus \\
\hline [20] & Phansalkar S & 2010 & Usability & Targeted review \\
\hline$[21]$ & Pelayo S & 2011 & Usability & $\begin{array}{l}\text { Targeted review \& analysis of cognitive and } \\
\text { collaborative tasks }\end{array}$ \\
\hline [22] & Zachariah M & 2011 & $\begin{array}{l}\text { Development of a usability evaluation } \\
\text { instrument }\end{array}$ & $\begin{array}{l}\text { Phansalkar et al.s' review and feedback from } \\
\text { a preliminary evaluation }\end{array}$ \\
\hline [1] & Horsky J & 2012 & Usability & Targeted review \\
\hline [23] & Horsky J & 2013 & Usability & Targeted review \\
\hline [24] & Payne T & 2015 & Usability & Expert consensus \\
\hline
\end{tabular}




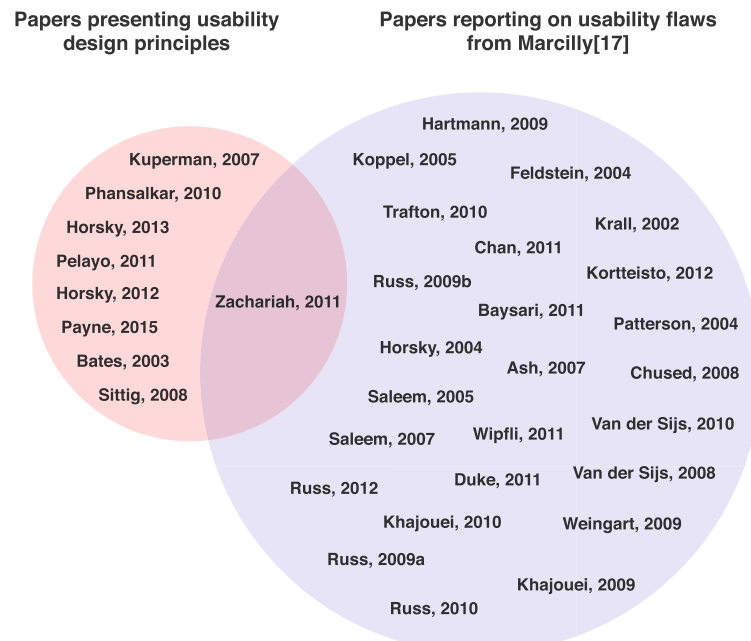

Fig. 3 Sets of papers analyzed. Left: the set of papers analyzed to establish a structured list of usability design principles for medication alerting systems. Right: the set of papers analyzed to establish the list of usability flaws in medication alerting systems [17]

A. Improve the system's signal-to-noise ratio, in order to decrease the frequency of over-alerting. In addition to the drugs ordered, the alert strategy should take into account parameters such as the patient's clinical context or the clinician's specialty. Moreover, the system must provide tools to customize the knowledge implemented within it and to monitor alert overrides.

B. Support collaborative work, advocate a team approach, and make the system a team player. The alerting system must encourage collaboration between the healthcare professional managing medications (e.g. physicians, pharmacists and nurses). Overall, alerts must deliver the same information to all clinicians, even if additional supplementary data can be presented as a function of the healthcare professional's role. The alerting system must help clinicians to understand how other healthcare professionals have already managed the alert.

C. Fit with clinicians' workflow and their mental model. The alerting system must comply with clinicians' needs and tasks. Alerts must be presented at the right moment in the decisionmaking process. Only the most severe alerts must interrupt the users; other alerts must be displayed more discreetly. Alerts must be concise, understandable and consistently structured so that users can easily find the relevant data. Once the alert has been satisfied, the clinicians must be able to resume their tasks easily.

D. Display relevant data within the alert. The system must provide clinicians with the information needed to make informed decisions. This includes the cause of the unsafe event (the medications involved), the description of the unsafe event, the severity/priority of the event, the mechanism of the interaction, the patient's clinical context, and evidence supporting the alert. Lastly, the system must suggest - but not impose - a means of remedying or monitoring the unsafe event.

E. Make the system transparent for the user. The alerting system must help clinicians to understand what the system can and cannot do and how it works, in order to prevent erroneous interpretation of its behavior. The user must have access to (i) the types of data that are checked, (ii) the formulas and rules applied, (iii) the list of the unsafe events that are targeted, and (iv) a description of the alerts' levels of severity.

F. Include actionable tools within the alert. The alert must provide several tools that help clinicians to easily and quickly translate their alert-informed clinical decision into actions: for example, buttons to modify/cancel/discontinue an order or override the alert, to order actions for monitoring an event, and to provide patient education. Other tools are recommended for managing the alert: pulling up the alert at a later time, sending the alert into a clinical note, removing the alert for a patient, and gaining access to the patient's medical records.

The final list of summarized usability design principles is given in Fig. 4. Table 2 provides a detailed version of the principles and corresponding flaws.

\section{Discussion}

\section{Answers to study questions}

The present study sought primarily to provide a specific, comprehensive, structured list of usability design principles for the medication alerting systems implemented in hospital or primary care settings. The secondary objective was to pair this list with the set of documented usability flaws, assess the match between the usability flaws that are known and the existing design principles, obtain illustrations of the existing violations of the principles, and present evidence that not applying usability design principles may be detrimental.

A total of 60 specific usability design principles for medication alerting systems were identified and organized hierarchically around 6 meta-principles: (A) improve the signal-to-noise ratio, (B) support collaborative work, (C) fit the clinicians' workflow and their mental model, (D) display relevant data within the alert, (E) make the system transparent for the user, and (F) include actionable tools within the alert. The 


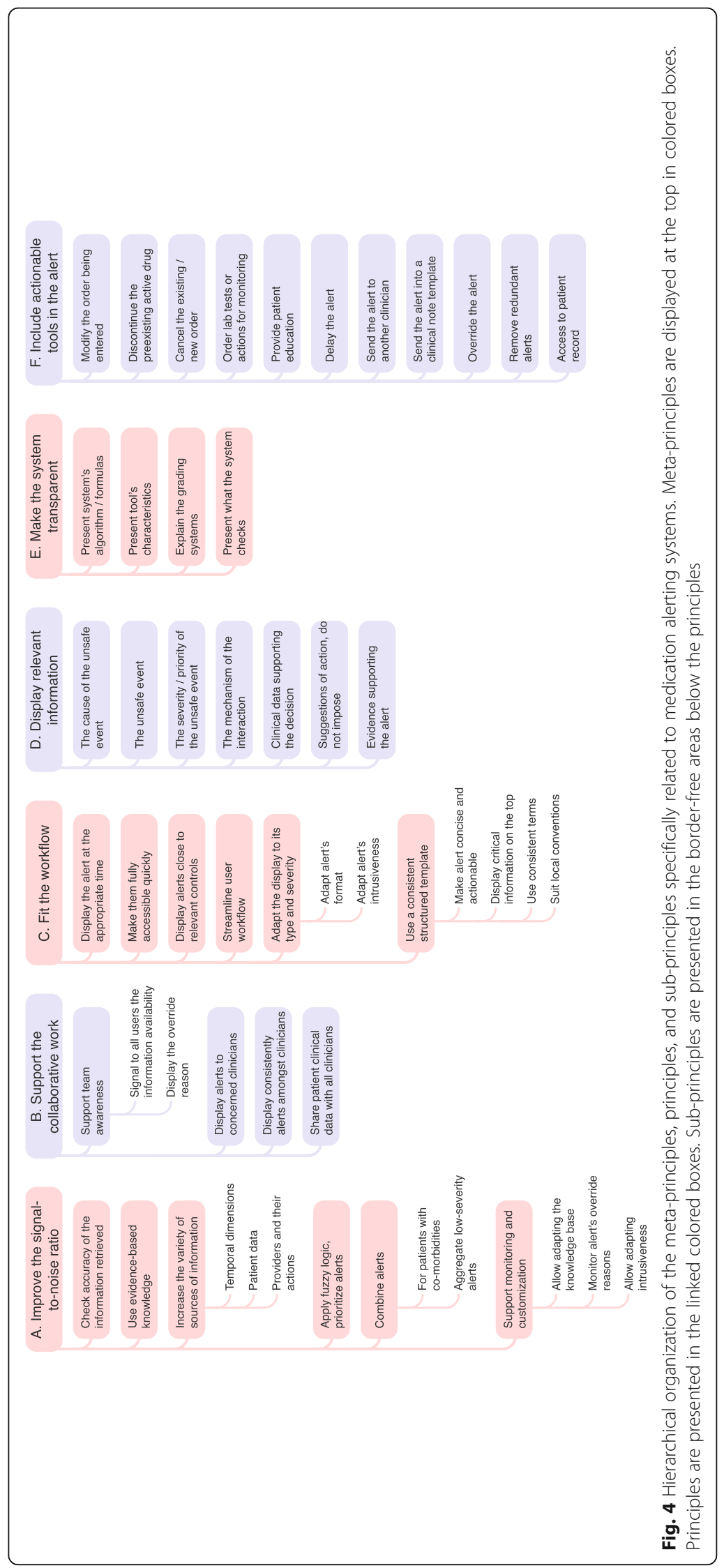




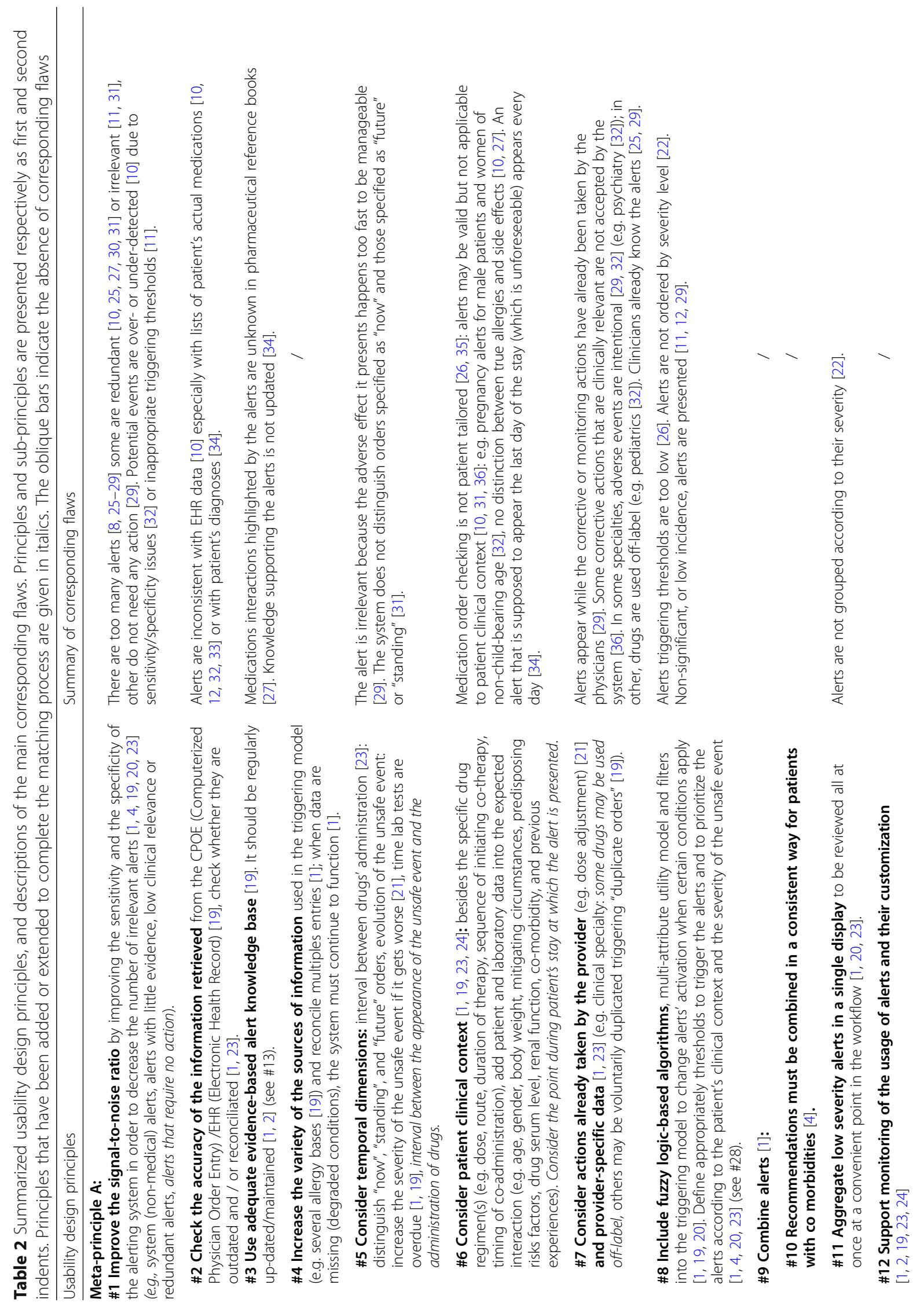




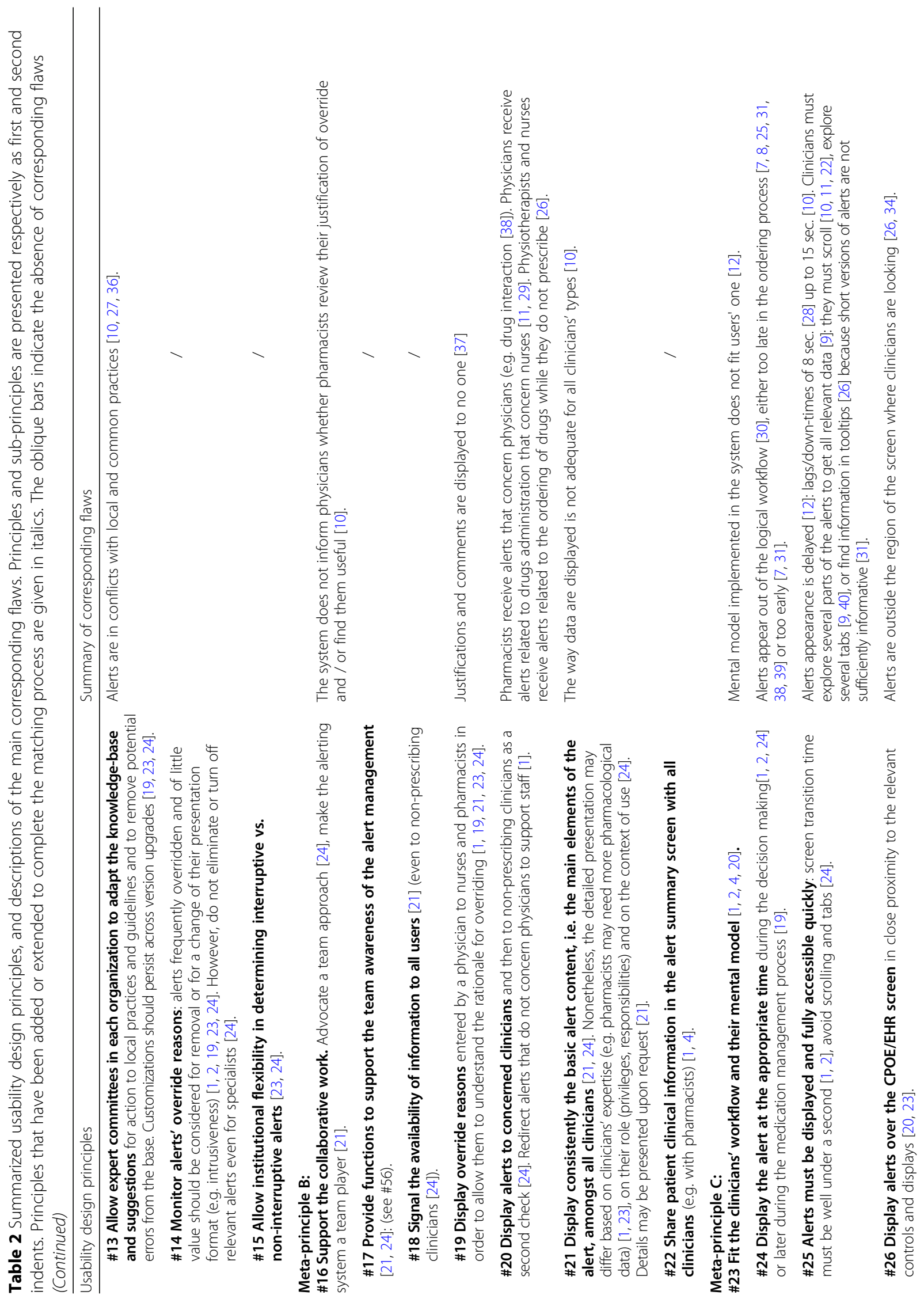




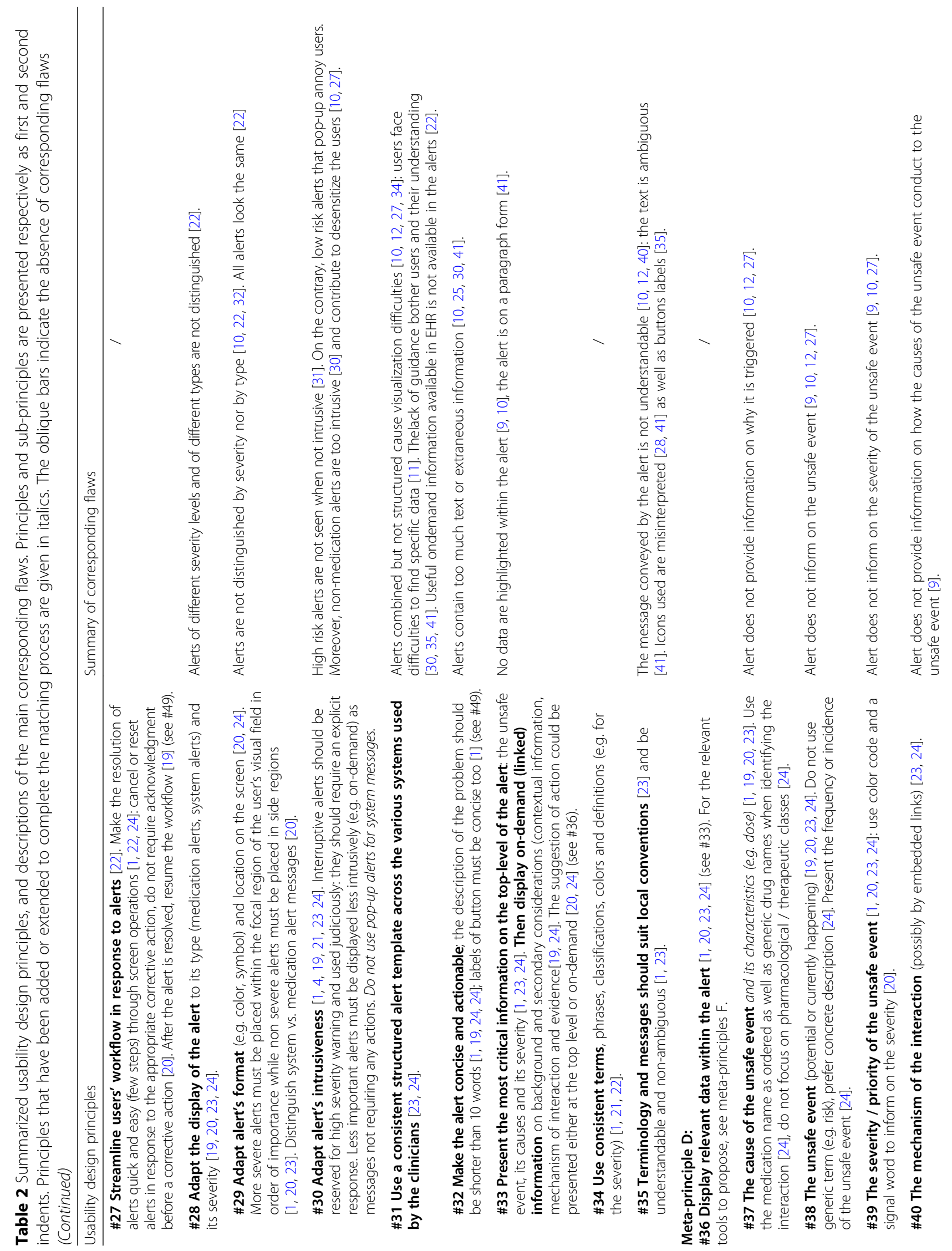




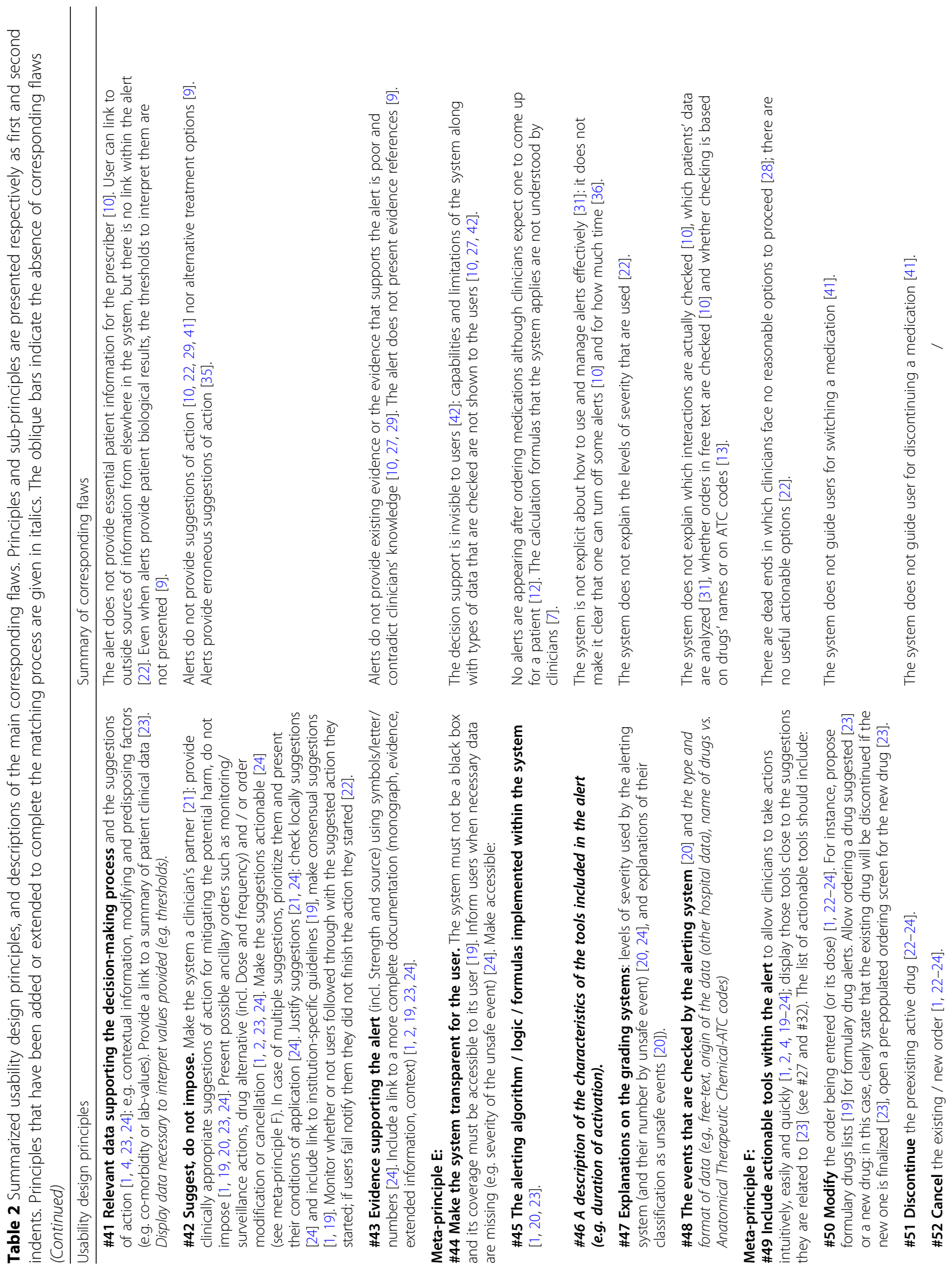




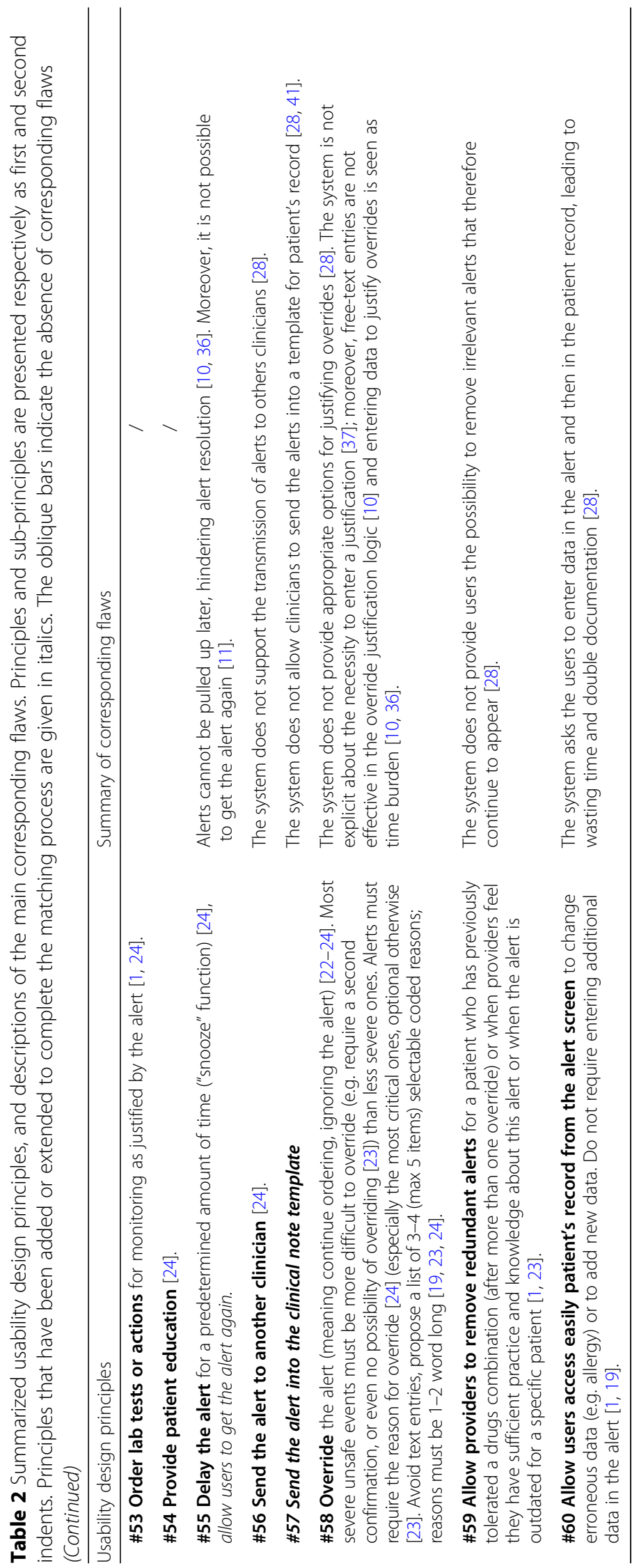


9 analyzed publications contributed to this list to different extents; we consider that the collation of several sets of usability design principles found in the literature expands the variety of topics represented in each individual set.

The match between the summarized usability design principles and the list of documented usability flaws was quite good: 34 principles were directly matched, and the context of application was extended for 9 principles. Nonetheless, 15 principles did not match any of the documented usability flaws. In view of the hierarchical organization of the principles, some principles are also not matched because their meta-principle or one or more of their sub-principles are matched - thus artificially reducing the quality of the match. We also identified limited gaps in the principles found in the literature; two new principles had to be created.

From a qualitative point of view, a few instances of usability flaws appear to contradict the corresponding usability design principles. For instance, some principles recommend including non-prescribers (e.g. pharmacists and nurses) in the alert management process, in order to promote collaboration between healthcare professionals (e.g. \#20). However, it has been reported that nurses are annoyed by medication alerts that interrupt their work [26]. The balance between promoting collaboration between healthcare professionals and not disrupting non-prescribers' tasks is delicate. Overall, instances of usability flaws must be used so that the corresponding design principles are not taken too literally.

\section{Study strengths}

The results of the present study represent an improvement with respect to the current literature. We did not change the principles extracted from the literature. By combining and summarizing the extracted principles, they are now clearly identified, listed, and organized hierarchically into a comprehensive, consistent, and structured hierarchy. Furthermore, the process of matching the principles to the usability flaws allows one to identifying evidence to show that not applying these principles has negative consequences. Each principle is now associated with the best available evidence of its violation. As far as we know, the present study is the first to have drawn up this type of list.

In addition to providing evidence, the matching process also provided concrete illustrations of violations of usability design principles. The illustrations may help people designing and evaluating alerting systems to identify the "usability mistakes" that should not be made or to catch these mistakes during the evaluation phases. In fact, the illustrations provide a clearer understanding of the design principles to be applied.

\section{Study limitations}

The retrieval of the usability design principles might have biased the representativeness of the principles and the flaws. We considered only publications reporting general sets of design principles, rather than evaluations giving system-specific usability recommendations. Grey literature was excluded. Moreover, most of the analysis was performed by one investigator, with a second investigator independently crosschecking the results. Together, these biases might have caused us to miss a few relevant principles. Consequently, the principles that we extended or created in the present study may have already been described in other publications (e.g. as system-specific recommendations on usability). Likewise, some usability flaws might have been missed during the systematic review [17] due to publication and reporting biases: it might have been possible to match principles not matched in the present study with usability flaws documented outside our review [17]. Despite these limitations, the match between the usability design principles and the usability flaws was quite good and ensured that the principles and flaws retrieved were representative. This good level of matching might be due (at least in part) to the inclusion of Zachariah's publication [22] and reports written by closely linked authors in both sets of publications (i.e. the set used to establish the list of principles and the set used to establish the list of flaws, e.g. $[4,20,23])$. In the present study, the risk of self-matching bias was considered to be acceptable because our objective was to obtain the most comprehensive possible list of design principles and corresponding flaws. On the contrary, not including a publication in one set because its authors had also worked on a publication included in the other set could have led us to ignore relevant usability design principles and/or usability flaws.

The frequency of appearance of the design principles was analyzed in order to establish the level of support for the design principles (i.e. the number of publications they were found in). However, we do not interpret this number as an indicator of which principles should be prioritized. Firstly, reporting and publishing biases and differences in the focus of the publications analyzed may have biased the frequency of appearance. Even without these biases, prioritizing the principles would imply that we are able to predict the severity of the consequences of the related usability flaws. However, the severity depends on many other factors, such as the system's other features and other flaws, and the context of use. This is one reason why most sets of design principles - whether developed for interactive systems (e.g. Nielsen's [43] and Scapin's [44] sets) or for a specific type of technology (e.g. the ones included in our analysis) - do not prioritize 


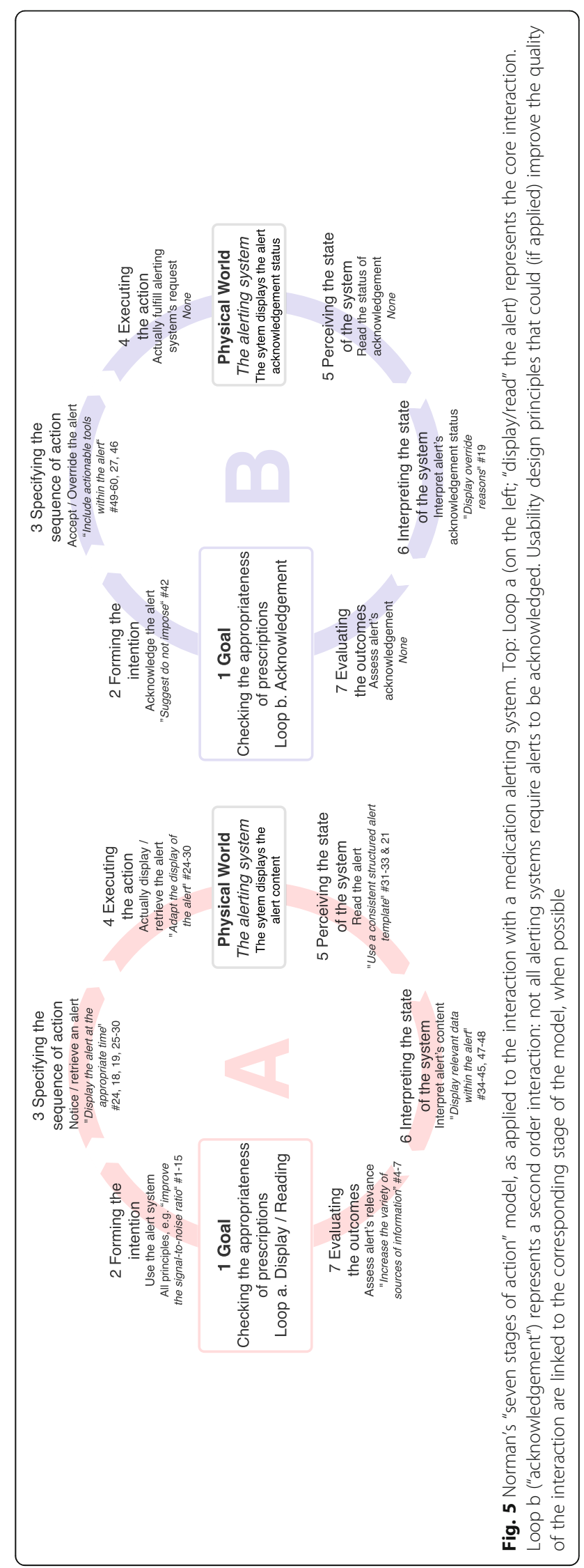


principles. Design principles can be prioritized by a person who is aware of the alerting system's characteristics and context of use.

In the present study, we addressed the evidence in favor of usability design principles by examining the violation of these principles. Evidence to suggest that applying design principles is beneficial has not yet been considered, due to reporting bias in the literature. Even though our present evidence is not based on instances of successful design, it may be convincing enough to persuade designers to apply usability design principles. Once researchers have begun to report on the positive usability characteristics of medication alerting system, the present analysis will have to be updated.

\section{The significance of the present results for a user interacting with a medication alerting system}

Usability design principles are related to various components of the alerting system: the triggering model, the knowledge implemented, the cognitive model implemented in the system, the information displayed, and the tools proposed within the alert. Applying these usability design principles might improve the clinician-alerting system interaction and the collaboration between clinicians. According to Norman's "seven stages of action" model [45], the user's interaction with a system encompasses two stages: the action stage translates a goal into an action sequence, and the evaluation stage compares the changes perceived in the world with the initial goal of the action (see Fig. 5). A clinician interacts with an alerting system in order to check the appropriateness of the prescriptions (step 1). Two "action and evaluation loops" may then be described. The main loop is "display/read" the alert. The second "acknowledgement" loop depends on the alerting system model; in some models, acknowledgment is not required.

For the "display/read" loop (loop a, Fig. 5, left), improving the overall usability of the alerting system by applying the whole set of design principles may facilitate the interaction and increase the clinician's intention to use the alerting system (step 2). More specifically, the whole "improving the signal-to-noise ratio" meta-principle may help to improve the relevance of the alerts, decrease alert fatigue, and thus increase the clinician's will to use the alerting system. In step 3, principles such as "signal the availability of information to all users" (\#18) and "display the alert at the appropriate time" (\#24) could make it easier to notice and retrieve alerts. In step 4, applying the "fit the clinician's workflow" meta-principle may help the clinician to display the alerts. Once alerts are displayed, clinicians have to read and interpret them (in steps 5 and 6). Applying the "use a consistent structured alert template", "display relevant data", and "make the system transparent" principles (\#31,
\#36, and \#44, respectively) may make the alerts more readable and help the clinicians to interpret them. Lastly, "extend[ing] the sources of information used in the triggering model" (\#4) may make it easier for clinicians to assess the alerts' relevance (step 7).

Once alerts are interpreted, physicians may have to acknowledge them (loop b, Fig. 5, right). Applying the "suggest - do not impose" principle (\#42) may increase the probability with which a clinician acknowledges the alert and perform corrective actions (step 2). Next, "includ[ing] actionable tools within the alerts" (\#49) may make it easier and quicker to specify and execute corrective actions (e.g. modify the order; step 3). If a physician overrides an alert and enters the reason why, "display[ing] override reason" (\#19) might help other clinicians to interpret the alert's acknowledgement status (step 6) and decide whether or not the alert has been properly assessed.

In summary, applying this set of usability design principles might improve both the action and evaluation stages of a user's interaction with the alerting system - mainly in the "display/read" loop but also in the "acknowledgement" loop. Some principles go beyond Norman's model, which relates to an individual's interaction with the alerting system and not interactions between clinicians or the clinicians' workflow. Adhering to the "fit the clinicians' workflow" meta-principle might decrease the risk of rejection. Moreover, if the "support collaborative work" meta-principle were to be applied, the alerting system could truly help clinicians to gain the same mental representation of the prescription being checked; this would help them to coordinate their actions and improve patient safety.

\section{Generalizability of the study}

The list of usability flaws used in the matching process might increase over time, depending on whether new publications report usability flaws. Moreover, technology evolves rapidly, and the related principles might change accordingly. For instance, the principles presented here are formulated for medication alerting systems implemented on laptop and/or desktop computers. However, as mobile health technologies are refined and expanded, alerting systems will be progressively installed on mobile devices. This might modify the applicability of the usability design principles listed here. It will therefore be essential to update this work regularly and take account of the latest trends and developments. However, the maintenance of this knowledge may be time-consuming, and represents a challenge for human factors specialists in the field of medical informatics. Manufacturers should be associated with this process.

Some design principles insist on the need for promoting collaboration between clinicians (\#16) but ignore the 
key person in the medication management process - the patient. Only principle \#54 mentions the patient (being able to "provide patient education"). However, as for other information technologies, the implementation of an alerting system changes the nature of the patient-clinician interaction [46]. It is important to ensure that poor usability has not damaged the patient-clinician interaction. On the contrary, increased usability should underpin patient-clinician discussion, empower the patient [47], and ensure that care remains patient-centered. The current literature on the usability of medication alerting systems does not consider the patient as a stakeholder in medication management. Future research on the usability of medication alerting system should integrate patients as stakeholders in medication management, so as to adapt or extend usability design principles to their specific features.

Although the structured design principles target only medication alerting systems implemented in hospital or primary care settings, some principles may be applied to other kinds of alerting systems. For instance, part of the "fit the clinicians' workflow" meta-principle could also be applied to laboratory result alerting systems. Nonetheless, the evidence that underpins the principles presented here is valid for medication alerting systems only.

In addition to the results, the method used to build this set of evidence-based usability design principles could also be applied to evidence-based usability design principles for other kinds of technology. However, this method is very time-consuming, and requires in-depth knowledge of the usability of the technology in question if the data are to be analyzed correctly.

\section{Turning the results into a usable, practical tool for designers and evaluators}

The present set of evidence-based usability design principles for medication alerting systems must be made accessible to and usable by designers and evaluators. At present, the principles are presented as a printable table (Table 2) that might not be ideal for optimal use. We intend to use the table to develop tools that present the evidence-based knowledge in a way that suits the needs of the various system designers and evaluators (usability experts, computer scientists, etc.) in various contexts of use (design, evaluation, procurement processes, etc.). With that aim in mind, we have started to identify the needs of medication alerting system designers and evaluators [48]. Accordingly, we developed (i) a checklist that measure the appropriate use of evidence-based principles in the design of medication alerting systems, and (ii) a set of interactive design instructions illustrated by visual representations of good and bad usability practices, in order to help designers make informed design decisions.
This list of usability design principles should help designers to make evidence-based usability design decisions. Nonetheless, and even though we believe that the list is helpful, it is not intended to be used as a stand-alone system or to replace the requirement for expertise in usability and design. Firstly, the present list does not include general design principles for unspecified interactive systems; it must therefore be used in combination with sets of general usability design principles for interactive systems (e.g. $[43,44])$. Secondly, several principles require insights into the users' cognitive tasks and their decision-making processes in order to adjust (for instance) an alert's format and the moment at which it appears (e.g. \#24). Hence, work system and cognitive work analyses [49] must be performed so the principles are applied in an optimal way. Thirdly, principles moderate each other; they must not be applied alone or in an unquestioning manner. Human factors specialists and designers must use their expertise to determine which principles must be applied and how they must be applied, given the characteristics of the alerting system and the setting in which it is implemented in (hospital vs. primary care, for example). In summary, this structured list of usability design principles must be used as a support for expertise and not as a substitute for it.

Applying some of the principles listed here may present specific technical and organizational challenges when seeking to tailor alerts. For instance, the "prioritize the alerts according to patient's clinical context and the severity of the unsafe event" (\#8) principle requires access to valid data on the patient's clinical context, stay, and treatment. However, these data are often not standardized or structured enough to be used in the alerting system's set of rules [50]. Further research is needed to overcome these challenges.

Ultimately, presenting designers and evaluators with evidence-based knowledge may help to decrease the occurrence of unforeseen and potentially harmful usability-induced use errors. Nonetheless, one must be aware that improving the usability of an existing system or ensuring that the usability of a system under development is optimal is no guarantee of success. Other issues arising during the development of a medication alerting system (e.g. an error-ridden knowledge base, a poor implementation process, unsuitable settings, etc.) can ruin even optimal levels of usability. Even though it is necessary to consider usability during the design, development, and evaluation of medication alerting systems, one must never neglect the relevant technical, social, and managerial factors that also contribute to the system's success or failure.

\section{Conclusions}

In the present study, we developed an evidence-based, structured, specific, comprehensive list of usability design principles for medication alerting systems, and then 
illustrated them with the corresponding usability flaws. This list should help designers and usability experts to gain a better understanding of usability design principles. We expect that the list can be used during the design and evaluation processes of medication alerting systems, in order to prevent usability issues that could have a counterproductive impact on clinicians (e.g. alert fatigue) and potentially harmful outcomes for patients (e.g. errors in medication dosing). Although operational barriers may complicate the deployment and maintenance of the evidence-based usability design principles presented in the present study, our results show that the approach is feasible. Indeed, our approach could be transferred to other health information technologies for the generation of specific lists of evidence-based usability design principles. In this way, designers and evaluators could be provided with tools to help them avoid usability design issues in health information technology and thus decrease the likelihood of unforeseen and potentially harmful usability-induced use errors.

\section{Additional files}

Additional file 1: Appendix 1. List of usability design principles identified in the 9 papers and the corresponding usability design principles summarized (for definitions, please refer to Table 2). (DOCX 248 kb)

Additional file 2: Appendix 2. The 9 papers' contributions to the summarized principles. Crosses show that a given principle is mentioned in a paper. The right-hand-most column gives the number of papers mentioning a given principle. The bottom two rows present the number of principles mentioned by each paper and the proportion of the full list of principles mentioned by each paper. It should be noted that the percentages are based on the 58 principles summarized in step 1. Principles \#46 and \#57 were created after the matching process and therefore were not included here. (DOCX $56 \mathrm{~kb}$ )

Additional file 3 Appendix $\mathbf{3}$. Results of the matching between instances of usability flaws (from Marcilly et al. [17]) and the usability design principles summarized in the present study. (DOCX $82 \mathrm{~kb}$ )

\section{Abbreviations}

ATC: Anatomical Therapeutic Chemical; CPOE: Computerized Physician Order Entry; EHR: Electronic Health Record

\section{Acknowledgements}

The authors would like to thank the staff at the University of Lille 2 library for their very efficient work in retrieving the required publication. The author would like to thank Melissa Baysari for her feedback on the wording of Table 2, Emmanuel Castets and Pierre-François Gautier for the figures' design. Finally, the authors would like to thank the reviewers and editors for their constructive comments.

\section{Availability of data and materials}

The dataset(s) supporting the conclusions of this article is(are) included within the article (and its additional file(s)).

\section{Authors' contributions}

RM designed the study, retrieved the data, performed the analysis and wrote the paper. EA helped to design the study, provided methodological support and supported the writing of the paper by reading it several times and providing advice to improve the report of the study. ER supported the writing of the paper by reading it several times and validating it. Additionally, ER checked English spelling and grammar. JN provided a methodological support and supported the writing of the paper by reading it several times and providing advice to improve the report of the study. MCBZ retrieved the data, performed the analysis and supported the writing of the paper by reading it several times and providing advice to improve the report of the study.

All authors approved the present version of the paper.

Ethics approval and consent to participate

Not applicable.

\section{Competing interests}

The authors declare that they have no competing interests.

\section{Publisher's Note}

Springer Nature remains neutral with regard to jurisdictional claims in published maps and institutional affiliations.

\section{Author details}

'Univ. Lille, INSERM, CHU Lille, CIC-IT / Evalab 1403 - Centre d'Investigation clinique, EA 2694, F-59000 Lille, France, Maison Régionale de la Recherche Clinique, 6 rue du professeur Laguesse, 59000 Lille France. ${ }^{2}$ Institute of Medical Informatics, UMIT - University for Health Sciences, Medical Informatics and Technology, 6060 Hall in Tirol, Austria. ${ }^{3}$ eHealth Services Research Group, School of Engineering and ICT, University of Tasmania, Private Bag 87, Hobart, Tasmania 7001, Australia. ${ }^{4}$ General Electric Healthcare Partners, 92772, Boulogne Billancourt cedex, France.

Received: 22 June 2017 Accepted: 23 May 2018

Published online: 24 July 2018

\section{References}

1. Horsky J, Schiff GD, Johnston D, Mercincavage L, Bell D, Middleton B. Interface design principles for usable decision support: a targeted review of best practices for clinical prescribing interventions. J Biomed Inform. 2012; 45:1202-16.

2. Bates DW, Kuperman GJ, Wang S, Gandhi T, Kittler A, Volk L, Spurr C, Khorasani R, Tanasijevic M, Middleton B. Ten commendments for effective clinical decision support: making the practice of evidence-based medicine a reality. J Am Med Inform Assoc. 2003:10:523-30.

3. Jaspers MW, Smeulers M, Vermeulen H, Peute LW. Effects of clinical decision-support systems on practitioner performance and patient outcomes: a synthesis of high-quality systematic review findings. J Am Med Inform Assoc. 2011;18:327-34.

4. Sittig DF, Wright A, Osheroff JA, Middleton B, Teich JM, Ash JS, Campbell E, Bates DW. Grand challenges in clinical decision support. J Biomed Inform. 2008:41:387-92.

5. Campbell EM, Guappone KP, Sittig DF, Dykstra RH, Ash JS. Computerized provider order entry adoption: implications for clinical workflow. J Gen Intern Med. 2009;24:21-6.

6. Marcilly R, Ammenwerth E, Roehrer E, Pelayo S, Vasseur F, Beuscart-Zephir MC. Usability flaws in medication alerting systems: impact on usage and work system. Yearb Med Inform. 2015;10:55-67.

7. Horsky J, Kaufman DR, Patel VL. Computer-based drug ordering: evaluation of interaction with a decision-support system. Stud Health Technol Inform. 2004:107:1063-7.

8. Ash JS, Sittig DF, Campbell EM, Guappone KP, Dykstra RH. Some unintended consequences of clinical decision support systems. AMIA Annu Symp Proc. 2007:26-30.

9. Duke JD, Bolchini DA. Successful model and visual design for creating context-aware drug-drug interaction alerts. AMIA Annu Symp Proc. 2011: 339-48.

10. Russ AL, Zillich AJ, McManus MS, Doebbeling BN, Saleem JJ. Prescribers' interactions with medication alerts at the point of prescribing: a multimethod, in situ investigation of the human-computer interaction. Int J Med Inform. 2012;81:232-43.

11. van der Sijs H, van Gelter T, Vulto A, Berg M, Aarts J. Understanding handling of drug safety alerts: a simulation study. Int J Med Inform. 2010;79:361-9.

12. Russ AL, Saleem JJ, McManus MS, Zillich AJ, Doebbling BN. Computerized medication alerts and prescriber mental models: observing routine patient care. Proc Human Factors Ergon Soc Annu Meet. 2009:53:655-9.

13. Hartmann Hamilton AR, Anhoj J, Hellebek A, Egebart J, Bjorn B, Lilja B. Computerised physician order entry (CPOE). Stud Health Technol Inform. 2009;148:159-62. 
14. International Standardization Organization Ergonomics of human system interaction - part 210: human centered design for interactive systems (rep N ๑9241-210). Geneva: international standardization Organization; 2010.

15. Marcilly R, Peute L, Beuscart-Zephir MC. From usability engineering to evidence-based usability in health IT. Hat evidence supports the use of computerized alerts and prompts to improve clinicians' prescribing behavior? Stud Health Technol Inform. 2016;222:126-38.

16. Marcilly R, Beuscart-Zephir MC, Ammenwerth E, Pelayo S. Seeking evidence to support usability principles for medication-related clinical decision support (CDS) functions. Stud Health Technol Inform. 2013;192:427-31.

17. Marcilly R, Ammenwerth E, Vasseur F, Roehrer E, Beuscart-Zephir MC. Usability flaws of medication-related alerting functions: a systematic qualitative review. J Biomed Inform. 2015;55:260-71.

18. Nielsen J. Enhancing the explanatory power of usability heuristics. CHI '94 proceedings of the SIGCHI conference on human factors in. Comput Syst. 1994:152-8.

19. Kuperman GJ, Bobb A, Payne TH, Avery AJ, Gandhi TK, Burns G, Classen DC, Bates DW. Medication-related clinical decision support in computerized provider order entry systems: a review. J Am Med Inform Assoc. 2007;14: 29-40.

20. Phansalkar S, Edworthy J, Hellier E, Seger DL, Schedlbauer A, Avery AJ, Bates DW. A review of human factors principles for the design and implementation of medication safety alerts in clinical information systems. J Am Med Inform Assoc. 2010;17:493-501.

21. Pelayo S, Marcilly R, Bernonville S, Leroy N, Beuscart-Zephir MC. Human factors based recommendations for the design of medication related clinical decision support systems (CDSS). Stud Health Technol Inform. 2011; 169:412-6.

22. Zachariah M, Phansalkar S, Seidling HM, Neri PM, Cresswell KM, Duke J, Bloomrosen M, Volk LA, Bates DW. Development and preliminary evidence for the validity of an instrument assessing implementation of human-factors principles in medication-related decision-support systems-I-MeDeSA. J Am Med Inform Assoc. 2011;18(Suppl 1):162-72.

23. Horsky J, Phansalkar S, Desai A, Bell D, Middleton B. Design of decision support interventions for medication prescribing. Int J Med Inform. 2013;82: 492-503.

24. Payne $T H$, Hines LE, Chan RC, Hartman S, Kapusnik-Uner J, Russ AL, et al. Recommendations to improve the usability of drug-drug interaction clinical decision support alerts. J Am Med Inform Assoc. 2015;22:243-1250.

25. Baysari MT, Westbrook Jl, Richardson KL, Day RO. The influence of computerized decision support on prescribing during ward-rounds: are the decision-makers targeted? J Am Med Inform Assoc. 2015;18:754-9.

26. Kortteisto T, Komulainen J, Makela M, Kunnamo I, Kaila M. Clinical decision support must be useful, functional is not enough: a qualitative study of computer-based clinical decision support in primary care. BMC Health Serv Res. 2012;12:349-57.

27. Russ AL, Zillich AJ, McManus MS, Doebbeling BN, Saleem JJA. Human factors investigation of medication alerts: barriers to prescriber decisionmaking and clinical workflow. AMIA Annu Symp Proc. 2009:548-52.

28. Saleem JJ, Patterson ES, Militello L, Render ML, Orshansky G, Asch SM. Exploring barriers and facilitators to the use of computerized clinical reminders. J Am Med Inform Assoc. 2005;12:438-47.

29. van der Sijs H, Aarts J, van Gelter T, Berg M, Vulto A. Turning off frequently overridden drug alerts: limited opportunities for doing it safely. J Am Med Inform Assoc. 2008;15:439-48.

30. Feldstein A, Simon SR, Schneider J, Krall M, Laferriere D, Smith DH, Sittig DF, Soumerai SB. How to design computerized alerts to safe prescribing practices. Jt Comm J Qual Saf. 2004;30:602-13.

31. Krall MA, Sittig DF. Clinician's assessments of outpatient electronic medical record alert and reminder usability and usefulness requirements. Proc AMIA Symp. 2002:400-4.

32. Weingart SN, Massagli M, Cyrulik A, Isaac T, Morway L, Sands DZ, Weissman JS. Assessing the value of electronic prescribing in ambulatory care: a focus group study. Int J Med Inform. 2009;78:571-8.

33. Russ AL, Saleem JJ, McManus MS, Frankel RM, Zillich AJ. The workflow of computerized medication ordering in primary care is not prescriptive. Proc Human Factors Ergon Soc Annu Meet. 2010;54:840-4.

34. Wipfli R, Betrancourt M, Guardia A, Lovis CA. Qualitative analysis of prescription activity and alert usage in a computerized physician order entry system. Stud Health Technol Inform. 2011;169:940-4.
35. Khajouei R, Peek N, Wierenga PC, Kersten MJ, Jaspers MW. Effect of predefined order sets and usability problems on efficiency of computerized medication ordering. Int J Med Inform. 2010;79:690-8.

36. Patterson ES, Nguyen AD, Halloran JP, Asch SM. Human factors barriers to the effective use of ten HIV clinical reminders. J Am Med Inform Assoc. 2004;11:50-9.

37. Chused AE, Kuperman GJ, Stetson PD. Alert override reasons: a failure to communicate. AMIA Annu Symp Proc. 2008:111-5.

38. Koppel R, Metlay JP, Cohen A, Abaluck B, Localio AR, Kimmel SE, Strom BL. Role of computerized physician order entry systems in facilitating medication errors. JAMA. 2005;293:1197-203.

39. Khajouei R, de Jongh D, Jaspers MW. Usability evaluation of a computerized physician order entry for medication ordering. Stud Health Technol Inform. 2009;150:532-6.

40. Saleem JJ, Patterson ES, Militello L, Anders S, Falciglia M, Wissman JA, Roth EM, Asch SM. Impact of clinical reminder redesign on learnability, efficiency, usability, and workload for ambulatory clinic nurses. J Am Med Inform Assoc. 2007;14:632-40.

41. Trafton J, Martins S, Michel M, Lewis E, Wang D, Combs A, Scates N, Tu S, Goldstein MK. Evaluation of the acceptability and usability of a decision support system to encourage safe and effective use of opioid therapy for chronic, noncancer pain by primary care providers. Pain Med. 2010;11:575-85.

42. Chan J, Shojania KG, Easty AC, Etchells EE. Usability evaluation of order sets in a computerised provider order entry system. BMJ Qual Saf. 2011;20:932-40.

43. Nielsen J. Usability Engineering Boston: Academic Press; 1993.

44. Scapin DL, Bastien JMC. Ergonomic criteria for evaluating the ergonomic quality of interactive systems. Behav Inf Technol. 1997;6:220-31.

45. Norman DA. The Design of Everyday Things. New-York: Basic Book; 1988.

46. Pearce C. Computers, patients, and doctors-theoretical and practical perspectives. In: Shachak A, Borycki EM, Reis SP, editors. Health Professionals' Education in the Age of Clinical Information Systems, Mobile Computing and Social Networks: Academic Press, Elsevier; 2017.

47. Náfrádi L, Nakamoto K, Schulz PJ. Is patient empowerment the key to promote adherence? A systematic review of the relationship between self-efficacy, health locus of control and medication adherence. PLoS One. 2017;12

48. Marcilly R, Monkman H, Villumsen S, Kaufman D, Beuscart-Zéphir M-C. How to present evidence-based usability design principles dedicated to medication-related alerting systems to designers and evaluators? Results from a workshop. Stud Health Technol Inform. 2016;228:609-13.

49. Vicente KJ. Cognitive work analysis. Mahwah, NJ: Lawrence Erlbaum Associates: 1999.

50. Riedmann D, Jung M, Hackl WO, Stuhlinger W, van der SH, Ammenwerth E. Development of a context model to prioritize drug safety alerts in CPOE systems. BMC Med Inform Decis Mak. 2011;11:35.

\section{Ready to submit your research? Choose BMC and benefit from:}

- fast, convenient online submission

- thorough peer review by experienced researchers in your field

- rapid publication on acceptance

- support for research data, including large and complex data types

- gold Open Access which fosters wider collaboration and increased citations

- maximum visibility for your research: over $100 \mathrm{M}$ website views per year

At BMC, research is always in progress.

Learn more biomedcentral.com/submissions 